01.1

\title{
Гетероструктура на основе гибрида даймондена и графена
}

\author{
() М.С. Чекулаев, С.Г. Ястребов \\ Физико-технический институт им. А.Ф. Иоффе РАН, Санкт-Петербург, Россия \\ E-mail: mchs89@mail.ioffe.ru
}

Поступило в Редакцию 4 сентября 2018 г.

В окончательной редакции 4 февраля 2019г.

Принято к публикации 4 фревраля 2019г.

Предложены идея гетероструктуры, представляющей собой гибрид даймондена и графена, и принцип построения таких гибридов на основе наблюдательных астрофизических данных. Построена модель, выполнены оптимизация геометрии и оценка стабильности молекулы universal force field и $a b$ initio методами.

DOI: 10.21883/PJTF.2019.08.47623.17516

Большинство из известных аллотропных модификаций углерода может существовать в виде частиц, инкапсулированных в матрицу аморфного углерода, и родственных аллотропных модификациях, таких как аморфный „алмазоподобный“ углерод. О прямом наблюдении с помощью метода просвечивающей электронной микроскопии нанокластеров алмаза, инкапсулированных в матрицу аморфного углерода, свидетельствуют, например, данные работ [1,2], а фрагментов графена - данные [3]. Более того, из анализа данных ИК-спектроскопии межзвездной среды известно, что наноразмерные кластеры, связанные $s p^{3}$-гибридизацией, могут существовать в межзвездной среде совместно с полициклическими ароматическими углеводородами, что следует из данных [4].

Кроме того, в аморфном углероде и межзвездной среде могут существовать молекулы, представляющие собой гибрид кластера, одна часть атомов которого связана $s p^{3}$-гибридизацией, а другая $-s p^{2}$-гибридизацией. В литературе упоминаются такие гибридные молекулы. Например, в [5] речь идет о пентаграфене, который представляет собой лист углерода, составленного из одних лишь пятиугольников. Однако рассматриваемой в данной работе структурой является фрагмент единичной алмазной плоскости направления (111) (даймонден, также известен в литературе как графан [6]), окруженный фрагментом единичной плоскости графита (графен), в отличие от работы [7], в которой была рассмотрена граница раздела этих фрагментов. Для компенсации избытка электронов на оборванных связях к ним может присоединяться водород: по одному атому водорода на каждый атом даймондена и по одному атому водорода на каждое краевое состояние фрагмента графена.

Из общих соображений следует ожидать, что такой гибрид будет устойчивым, как это имеет место, например, в случае букидаймонда [8]. Исследование квазидвумерных углеродных структур и фазовых переходов графен-алмазные многослойные пленки с помощью эмпирических потенциалов или в рамках теории функционала плотности [9-12] дает возможность изучать свойства таких молекул при отсутствии возможности воспроизведения их в лабораторных условиях.
В настоящей работе приведены результаты математического моделирования и геометрической оптимизации гетеромолекулы на основе такого гибрида фрагмента даймондена и фрагмента графена.

С целью выяснения числа атомов, входящих в кластер, мы обратились к лабораторному эксперименту и наблюдательным астрофизическим данным.

На рис. 1, $a$ представлен спектр эмиссии космического объекта Elias 1 [4]. Для спектроскопической идентификации спектральных линий воспользуемся данными [13], которые представлены на рис. $1, b$. В соответствии с данными работы [13] полосу эмиссии с частотой $2830 \mathrm{~cm}^{-1}$ можно приписать колебаниям двух атомов водорода, связанных с атомом углерода, охваченным $s p^{3}$-гибридизацией $\left(\mathrm{CH}_{2}\right)$. Частота $2920 \mathrm{~cm}^{-1}$ характерна для колебаний атома водорода, связанного с единичным атомом углерода, охваченным $s p^{3}$-гибридизацией $(\mathrm{CH})$. Отметим также, что частота $3035 \mathrm{~cm}^{-1}$ характерна для колебаний атома водорода, связанного с атомом углерода, охваченным $s p^{2}$-гибридизацией $(\mathrm{CH})$. Заметим, что значительная ширина компонент спектра поглощения [13] объясняет незначительное расхождение абсолютных значений частот в спектре космического объекта Elias1 (рис. 1,a) со значениями, представленными на рис. $1 b$. Полоса поглощения с частотой растяжения $s p^{3}$ для $\mathrm{CH}_{2}$-связей является наиболее интенсивной в спектре, что может быть следствием присутствия в межзвездной среде значительного количества небольших даймондоидов. Наличие в спектре полосы $3035 \mathrm{~cm}^{-1}$ СН может свидетельствовать о присутствии в межзвездной среде ароматических полициклических углеводородов. С другой стороны, наряду с даймондоидами и полициклическими ароматическими углеводородами в межзвездной среде может существовать их гибрид гетеромолекула или гетерокластер на основе атомов углерода и водорода.

С целью идентификации этой молекулы (кластера) интересно провести оптимизацию геометрии такой гетеромолекулы (гетерокластера), состоящей из двух частей, одна из которых связана $s p^{3}$-гибридизацией, а вторая $s p^{2}$-гибридизацией. Для оценки числа атомов углерода 

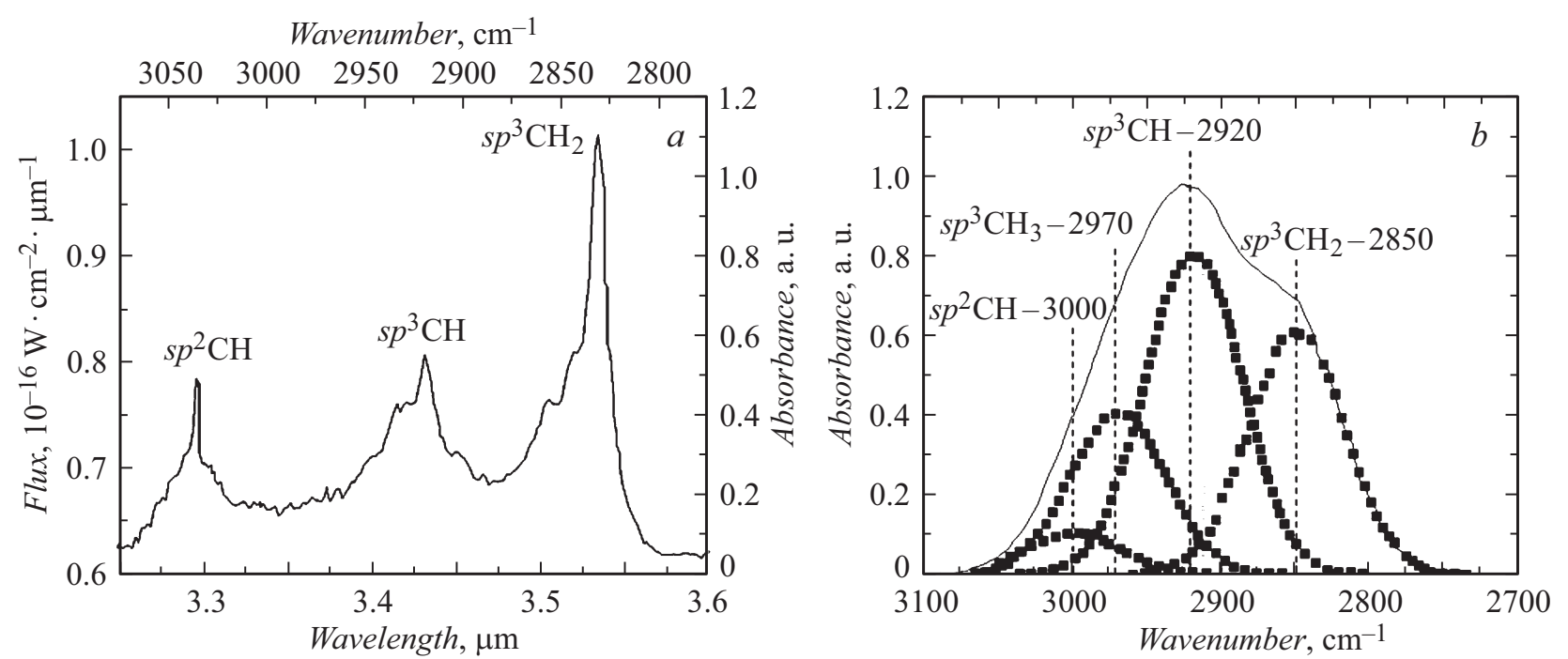

Рис. 1. $a$ - спектр поглощения космического объекта Elias 1 (данные работы [4]); $b$ - разложение пика $2900 \mathrm{~cm}^{-1}$ ИК-спектра алмазоподобных пленок (данные работы [13]).

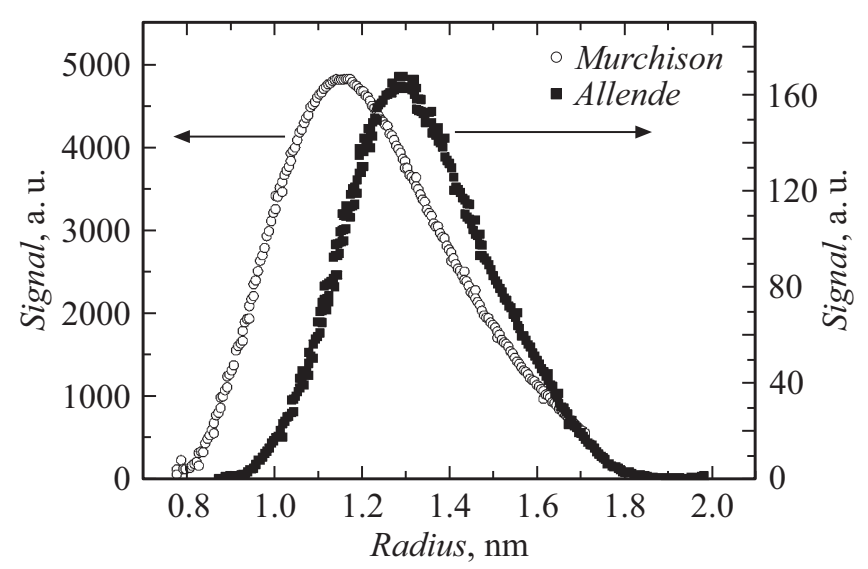

Pис. 2. Распределение наноалмазов в метеоритах Murchison и Allende в зависимости от размера (по данным работы [15]).

в такой молекуле обратим внимание на то, что в работе [14] приведены коэффициенты, связывающие интенсивность полос 2920 и $3035 \mathrm{~cm}^{-1}$ в спектре эмиссии с количеством атомов водорода согласно формуле

$$
I=A_{n} N_{\mathrm{H}}
$$

где $A_{n}-$ коэффициент пропорциональности, $N_{\mathrm{H}}-$ количество атомов водорода.

Соотношение атомов водорода, связанных с $s p^{2}-$ и $s p^{3}$-углеродом, найдем из отношения интенсивностей полос в спектре эмиссии космического объекта Elias 1 по формуле

$$
\frac{N_{\mathrm{H} s p^{3}}}{N_{\mathrm{H} s p^{2}}}=\frac{I_{\mathrm{H} s p^{3}}}{A_{n s p^{3}}} \frac{A_{n s p^{2}}}{I_{\mathrm{H} s p^{2}}},
$$

где $N_{\mathrm{H} s p^{3}}$ и $N_{\mathrm{H} s p^{2}}$ - количество водорода, связанного с $s p^{3}$ - и $s p^{2}$-углеродом соответственно, $I_{\mathrm{H} s p^{3}}$ и $I_{\mathrm{H} s p^{2}}-$ интенсивности полос на частоте 2920 и $3035 \mathrm{~cm}^{-1}$, а
$A_{n s p^{3}}$ и $A_{n s p^{2}}$ - коэффициенты пропорциональности для этих полос.

Подставив все данные в формулу (2), мы нашли соотношение атомов водорода между фазами, численное значение - 0.75. Поскольку речь идет об астрофизических объектах, для определения размера гетероструктуры обратимся к данным по метеоритному веществу. В работе [15] был выполнен анализ распределения наноалмазов в метеоритах Murchison и Allende в зависимости от размера (рис. 2). Видно, что радиусы наноалмазов в этих метеоритах находятся в интервале от 0.8 до $1.8 \mathrm{~nm}$.

В результате подбора $N_{\mathrm{H} s p^{3}}$ и $N_{\mathrm{H} s p^{2}}$ в качестве примера мы получили гетеромолекулу, состоящую из 16 атомов углерода с $s p^{3}$-гибридизацией, связанных с водородом, и 64 атомов углерода с $s p^{2}$-гибридизацией, 22 атома водорода связаны с краевыми атомами. Размер гетероструктуры $1.7 \times 1.8 \mathrm{~nm}$, что согласуется с данными работы по анализу метеоритов Murchison и Allende [15], а соотношение $\mathrm{CH}$-связей различной гибридизации согласуется с таковым у космического объекта Elias 1. Предварительная оптимизация была выполнена методом universal force field [16], реализованным в программе Avogadro [17]. Результат первичной оптимизации был подвергнут дополнительной проверке на предмет координационной устойчивости с помощью $a b$ initio метода (метода Хартри-Фока). В процессе геометрической оптимизации подтвердилась стабильность гетероструктуры. Результат оптимизации представлен на рис. 3, $a$. На рис. $3, b$ представлено распределение межатомных расстояний в гетероструктуре до оптимизации, на рис. 3,c - после оптимизации. Из рисунка следует, что во фрагменте графена произошло некоторое перераспределение длин связей: до оптимизации распределение связей во фрагменте графена представляло собой дельта-функцию, после оптимизации мы 

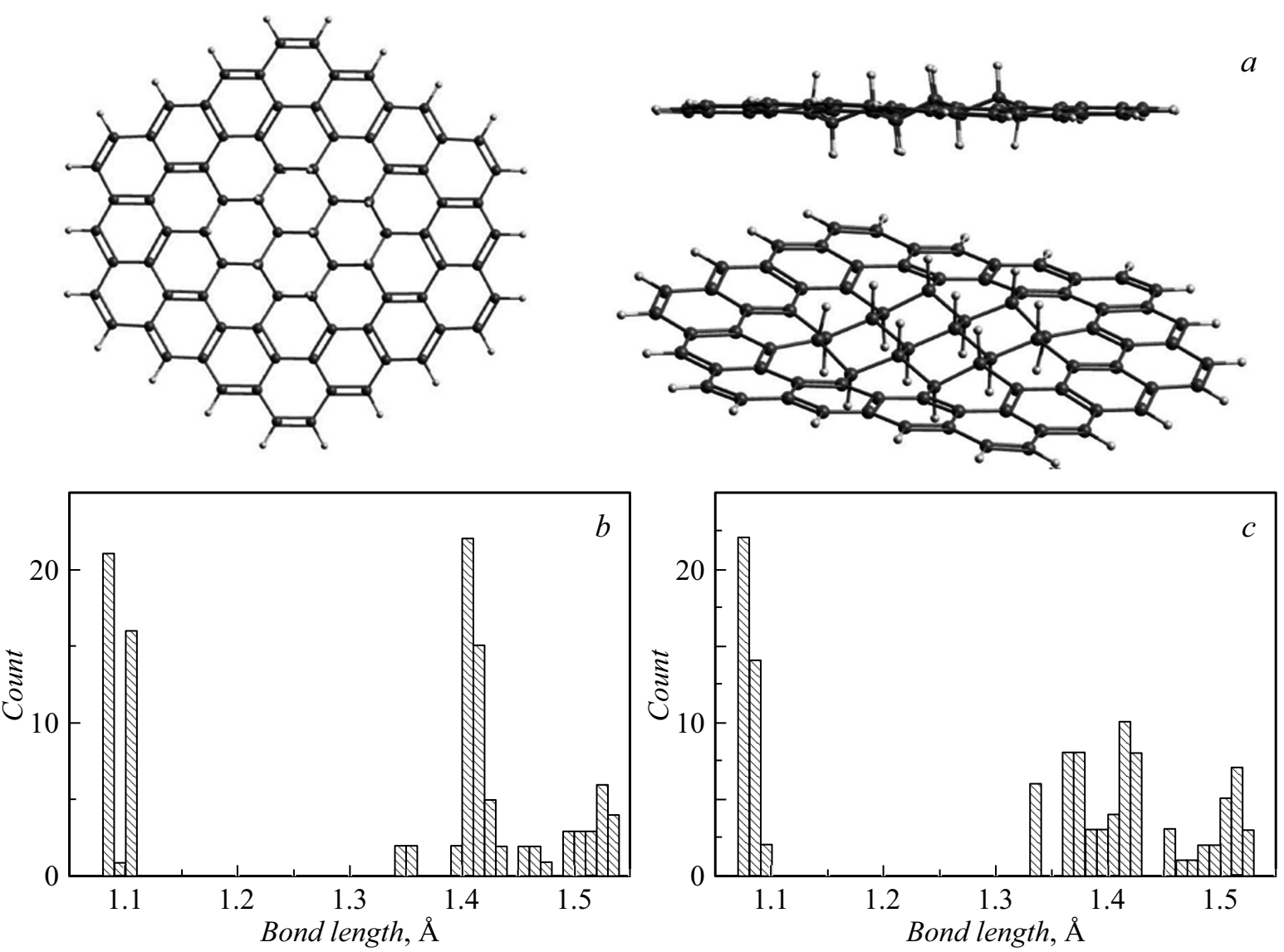

Рис. 3. $a-$ модель гетероструктуры после оптимизации; $b-$ распределение межатомных расстояний до оптимизации; $c-$ распределение межатомных расстояний после оптимизации.

видим однородное распределение связей в интервале $1.35-1.43 \AA$. Это связано с изменением межатомных расстояний краевых атомов углерода и атомов углерода, связывающих графен и даймонден.

Выполненные оценки устойчивости подобных структур могут оказаться важными для практического применения, например для генерации электромагнитного излучения. В этом смысле интерес может представлять двойная гетероструктура графен-даймонден. В случае протяженного фрагмента графена он может работать как контакт к активной области, в качестве которой может выступить наноалмаз.

Нами предложены идея гетероструктуры на основе гибрида даймондена и графена и принцип их построения для простого случая плоской геометрии.

Построена модель такой гетероструктуры для простого случая с минимальным числом атомов. Размеры построенной гетероструктуры хорошо согласуются с данными по анализу метеоритной субстанции, а соотношение СН-связей согласуется с таковым в спектрах эмиссии космических объектов. Результат оптимизации методом Хартри-Фока подтвердил устойчивость гетеромолекулы, что свидетельствует о возможности суще- ствования подобных гетеромолекул в межзвездной среде и возможности их синтеза в лабораторных условиях.

\section{Список литературы}

[1] Yastrebov S.G., Ivanov-Omskii V.I., Siklitsky V.I., Sitnikova A.A. // J. Non-Cryst. Solids. 1998. V. 227-230. Pt. 1. P. 622626. https://doi.org/10.1016/S0022-3093(98)00141-0

[2] Ivanov-Omskii V.I., Siklitsky V.I., Sitnikova A.A., Suvorova A.A., Tolmatchev A.V., Zvonariova T.K., Yastrebov S.G. // Phil. Mag. B. 1997. V. 78. N 6. P. 973-978. http://dx.doi.org/10.1080/01418639708243143

[3] Ivanov-Omskii V.I., Lodygin A.B., Sitnikova A.A., Suvorova A.A., Yastrebov S.G. // J. Chem. Vapor Deposition. 1997. V. 5. P. $198-206$.

[4] Geballe T.R. // ASP Conf. Ser. 1997. V. 122. P. 119.

[5] Zhang S., Zhou J., Wang Q., Chen X., Kawazoe Y., Jena P. // Proc. Natl. Acad. Sci. 2015. V. 112. N 8. P. 2372-2377.

[6] Sofo J.O., Chaudhari A.S., Barber G.D. // Phys. Rev. B. 2007. V. 75. N 15. P. 153401.

[7] Опенов Л.А., Подливаев А.И. // Письма в ЖЭТФ. 2009. T. 90. B. 6. C. 505-509.

[8] Raty J.-Y., Galli G., Bostedt C., van Buuren T.W., Terminelloet L.J. // Phys. Rev. Lett. 2003. V. 90. N 3. P. 037401. https://doi.org/10.1103/PhysRevLett.90.037401 
[9] Kvashnin A.G., Chernozatonskii L.A., Yakobson B.I., Sorokin P.B. // Nano Lett. 2014. V. 14. N 2. P. 676-681.

[10] Sorokin P.B., Kvashnin A.G., Zhu Z., Tomaánek D. // Nano Lett. 2014. V. 14. N 12. P. 7126-7130.

[11] Квашнин А.Г., Квашнина Ю.А., Антипина Л.Ю., Квашнина О.П., Сорокина Т.П., Сорокин П.Б. // Изв. вузов. Химия и хим. технология. 2014. Т. 57. В. 5. С. 71-74.

[12] Чернозатонский Л.А., Сорокин П.Б., Квашнин А.Г., Квашнин Д.Г. // Письма в ЖЭТФ. 2009. Т. 90. В. 2. С. 144148.

[13] Grill A., Patel V. // Appl. Phys. Lett. 1992. V. 60. N 17. P. 2089-2091.

[14] Titus E., Misra D.S., Sikder A.K., Tyagi P.K., Singh M.K., Misra A., Ali N., Cabral G., Neto V.F., Gracio J. // Diamond Relat. Mater. 2005. V. 14. N 14. P. 476-481.

[15] Lyon I.C. // Meteorit. Planet. Sci. 2005. V. 40. N 7. P. 981-987.

[16] Rappé A., Casewit C., Colwell K., Goddard III W., Skiff W. /I J. Am. Chem. Soc. 1992. V. 114. N 25. P. 10024-10035.

[17] Hanwell M.D., Curtis D.E., Lonie D.C., Vandermeersch T., Zurek E., Hutchison G.R. // J. Cheminformatics. 2012. V. 4. N 1. P. 17. 